\title{
Framingham cardiovascular disease risk scores and incident frailty: the English longitudinal study of ageing
}

\author{
Catharine R. Gale • Cyrus Cooper • Avan Aihie Sayer
}

Received: 3 June 2014 / Accepted: 17 July 2014 /Published online: 2 August 2014

(C) The Author(s) 2014. This article is published with open access at Springerlink.com

\begin{abstract}
Cross-sectional studies show that frailty is common in older people with cardiovascular disease. Whether older people at higher risk of developing cardiovascular disease are more likely to become frail is unclear. We used multinomial logistic regression to examine the prospective relation between Framingham cardiovascular disease risk scores and incidence of physical frailty or pre-frailty, defined according to the Fried criteria, in 1,726 men and women aged 60 to over 90 years from the English Longitudinal Study of Ageing who had no history of cardiovascular disease at baseline. Men and women with higher Framingham cardiovascular risk scores were more likely to become frail over the 4-year follow-up period. For a standard deviation higher score at baseline, the relative risk ratio (95\% confidence interval) for incident frailty, adjusted for sex and baseline frailty status, was $2.76(2.18,3.49)$. There was a significant association between Framingham cardiovascular risk score and risk of pre-frailty: $1.69(1.46,1.95)$. After further adjustment for other potential confounding factors, the relative risk ratios for frailty and pre-frailty were $2.15(1.68,2.75)$ and $1.50(1.29,1.74)$, respectively. The associations were unchanged after excluding
\end{abstract}

C. R. Gale $(\bowtie) \cdot$ C. Cooper $\cdot$ A. A. Sayer

MRC Lifecourse Epidemiology Unit, University of

Southampton,

Southampton SO16 6YD, UK

e-mail: crg@mrc.soton.ac.uk

C. R. Gale

Centre for Cognitive Ageing and Cognitive Epidemiology,

Department of Psychology, University of Edinburgh,

Edinburgh, UK incident cases of cardiovascular disease. Separate adjustment for each component of the risk score suggested that no single component was driving the associations between cardiovascular risk score and incident prefrailty or frailty. Framingham cardiovascular risk scores may be useful for predicting the development of physical frailty in older people. We now need to understand the biological mechanisms whereby cardiovascular risk increases the risk of frailty.

Keywords Frailty Cardiovascular risk · Cohort . Longitudinal study

\section{Introduction}

Frailty is a clinical syndrome observed in older people whose core feature is an increased vulnerability to stressors due to impairments in multiple, inter-related systems, decreased physiological reserves and a decline in the ability to maintain homeostasis (Bergman et al. 2007; Clegg et al. 2013). It is common (Gale et al. 2014a) and has numerous adverse consequences, including disability, falls, morbidity, hospitalization, institutionalization and death. Its causes are complex and likely to involve both biological and psychosocial mechanisms (Rockwood et al. 1994; Walston et al. 2006).

Cross-sectional surveys show that frailty and chronic disease frequently co-exist (Wong et al. 2010). One such disease whose relationship with frailty has been the focus of many studies is cardiovascular disease (CVD) 
(Afilalo et al. 2014; von Haehling et al. 2013). A systematic review of studies based on over 54,000 community-dwelling older people found that the odds ratios for prevalent frailty associated with CVD ranged from 2.7 to 4.1 (Afilalo et al. 2009) and that over a mean follow-up period of 6 years, the presence of CVD increased the risk of incident frailty by $50 \%$. These findings suggest that CVD risk may be predictive of future frailty and raise the possibility that multivariable risk factor algorithms for assessing CVD risk in primary care, such as that developed as part of the Framingham Heart Study (D'Agostino et al. 2008), might provide a method for identifying individuals currently free of CVD who are at increased risk of becoming frail. Longitudinal studies in the Whitehall II cohort have shown that in middleaged people without a history of stroke or heart disease, higher Framingham CVD risk scores are associated with poorer performance on objective tests of physical function (Elbaz et al. 2014) and with increased later risk of frailty (Bouillon et al. 2013), as defined by the Fried physical frailty phenotype (Fried et al. 2001). Whether such scores can predict future risk of frailty defined by the Fried phenotype in older people is unknown.

The English Longitudinal Study of Ageing (ELSA) is a large population-based sample of older men and women. We used these data to investigate the prospective relation between Framingham CVD risk scores and risk of incident frailty in men and women aged 60 to over 90 years (Marmot et al. 2013).

\section{Methods}

\section{Participants}

The data for this study come from the ELSA. The sample for ELSA was based on people aged $\geq 50$ years who had participated in the Health Survey for England in 1998, 1999 or 2001 (Steptoe et al. 2013). It was drawn by postcode sector, stratified by health authority and proportion of households in non-manual socioeconomic groups. A total of 11,392 people participated in wave 1 in 2002-2003. At wave 2 in 2004-2005 and at wave 4 in 2008-2009, core sample members (those in the household aged $\geq 50$ years who had taken part in wave 1) who had completed the main interview were invited to have a visit from a nurse that included measurements of physical function and blood pressure, anthropometry and blood sampling. Ethical approval was obtained from the Multicentre Research and Ethics Committee. Participants gave written informed consent.

Measures

Frailty

Maximum handgrip strength was measured three times on each side using a dynamometer; the best of these measurements was used for analysis. Height and weight were measured with a portable stadiometer and electronic scales, respectively. Body mass index (BMI) was calculated as weight (in kilograms)/height (in metres) ${ }^{2}$. Gait speed was assessed in participants aged 60 and over by measuring the time taken to walk a distance of $8 \mathrm{ft}$ at usual pace; the timed walk was repeated, and the mean of the two measurements was calculated. Participants responded to three questions about the frequency with which they did vigorous, moderate or mild exercise. We ranked the combinations of responses to these questions according to the amount and intensity of exercise involved to provide an estimate of usual physical activity. Symptoms of depression were assessed using the eightitem version of the Centre for Epidemiologic Studies Depression Scale (CES-D) (Steffick and The HRS working group 2000). We used these data, together with information on participants' weight at the initial survey, to derive an indicator of physical frailty at wave 2 (baseline) and at wave 4 in men and women aged $\geq 60$ years using the Fried criteria (Fried et al. 2001). These criteria define physical frailty as the presence of three or more of the following: unintentional weight loss, weakness, self-reported exhaustion, slow walking speed and low physical activity. We operationalized these criteria using definitions very similar to those used in the original phenotype of frailty studies (BandeenRoche et al. 2006; Fried et al. 2001): Weight loss was defined as either loss of $\geq 10 \%$ of body weight since the initial survey (for frailty at wave 2) or since wave 2 (for frailty at wave 4) or current BMI $<18.5 \mathrm{~kg} / \mathrm{m}^{2}$; weakness was defined as maximum grip strength in the lowest $20 \%$ of the distribution, after taking sex and BMI into account; exhaustion was considered present if the participant gave a positive response to either of the CES-D questions 'Felt that everything I did was an effort in the last week' or 'Could not get going in the last week'; slow walking speed was defined as a walking speed in the lowest $20 \%$ of the distribution, after taking account of sex and height; participants who did 
not perform the timed walk because they were unable to walk alone or had health restrictions were also categorised as having slow walking speed;and low physical activity was defined as physical activity in the lowest sex-specific $20 \%$ of the distribution.

\section{Framingham cardiovascular risk score at baseline}

The Framingham general cardiovascular risk score was developed for use in primary care to assess general cardiovascular risk and risk of individual events (coronary, cerebrovascular, peripheral arterial disease and heart failure) (D'Agostino et al. 2008). Its sex-specific algorithm is based on age, HDL and total cholesterol, systolic blood pressure, treatment for hypertension, smoking and diabetes.

Data on the risk score components was taken from the wave 2 examination. Blood samples were taken from all participants except those who were not willing to give written consent, those with clotting or bleeding disorders and those taking anti-coagulant drugs. Samples were assayed for total and HDL cholesterol, haemoglobin $\mathrm{A} 1 \mathrm{C}$ and in addition for fibrinogen and C-reactive protein at the Royal Victoria Infirmary, Newcastle-upon-Tyne, UK. Systolic blood pressure was measured three times using an Omron blood pressure monitor with the participant seated. We used the mean of these three values in our analysis. Participants were asked whether they were currently taking any medication for high blood pressure and about their current smoking status. Prevalent diabetes mellitus was defined based on reported doctor-diagnosed diabetes and/or use of diabetes medication, or a haemoglobin A1C level $\geq 6.5 \%$ (The International Expert Committee 2009).

\section{Covariates at baseline}

We chose cognitive function, household wealth and BMI as covariates because they could potentially confound any association between Framingham CVD risk score and later risk of frailty. Poorer cognition, lower socioeconomic status, as measured by household wealth, and higher BMI are independent predictors of frailty in both men and women in this cohort (Gale et al. 2014b), and have been shown to correlate with higher Framingham CVD risk scores (Elbaz et al. 2014; Kaffashian et al. 2013). Participants took tests of cognitive function as follows: Verbal memory (immediate and delayed recall of ten aurally presented nouns) was assessed using word lists developed for the US Health and Retirement Study (Ofstedal et al. 2005); prospective memory (remembering to do a specific task) and attention (letter cancellation task) were assessed using tests previously used in the MRC Cognitive Function and Ageing Study (Brayne et al. 1998); and executive function was assessed using a test of verbal (semantic) fluency (naming as many animals as possible in $60 \mathrm{~s}$ ) taken from the CAMCOG$\mathrm{R}$ (Roth et al. 1999). A total cognitive function score was calculated by summing scores on these tests. Socioeconomic status was indexed by total household wealth, including savings and investments, value of any property or business assets, net of debt, excluding pension assets. Household wealth has been identified as the most accurate indicator of long-term socioeconomic circumstances in ELSA (Banks et al. 2003).

In a previous study of this cohort, higher concentrations of the inflammatory markers fibrinogen and Creactive protein were significant predictors of incident frailty in women, though not in men (Gale et al. 2013). As higher levels of these biomarkers correlate with higher Framingham CVD risk scores (Noh et al. 2013; Park et al. 2010), they might potentially confound any link between these risk scores and frailty, at least in women. We therefore carried out a subset analysis in which we examined whether additional adjustment for concentrations of fibrinogen and C-reactive protein altered the association between Framingham CVD scores and risk of frailty.

Analytical sample

Of the 5,918 study members aged $\geq 60$ years who were interviewed at wave 2 in 2004-2005, 5,377 agreed to be visited by a nurse $(91 \%)$. Of these, 3,454 were interviewed and visited by a nurse at wave 4 in 20082009. We excluded prevalent cases of CVD at the wave 2 baseline (reported doctor diagnoses of angina, heart attack, congestive heart failure or stroke). The present analysis is based on 1,726 people who were free of CVD at baseline and had complete data on Framingham cardiovascular risk score and baseline covariates at wave 2 and frailty at wave 4 .

Statistical analysis

We used ANOVA and chi-square test to examine differences in baseline characteristics according to the presence of pre-frailty or frailty at follow-up. We used 
multinomial logistic regression to examine the relation between a standard deviation increase in Framingham CVD risk score at baseline and risk of incident prefrailty or frailty. We adjusted for sex and frailty status at baseline and then in addition for cognitive function, household wealth and BMI. In a subset of women only, we carried out an additional adjustment for the inflammatory biomarkers fibrinogen and C-reactive protein. In order to examine which components of the Framingham CVD risk score helped to explain its association with incident frailty, we examined the effect on the association of adjusting for each component (age, HDL and total cholesterol, systolic blood pressure, treatment for hypertension, smoking and diabetes) in turn.

All data were weighted to correct for sampling probabilities, non-response, and for differential sample loss between waves in order to make them more closely reflect the population from whom the ELSA sample was drawn. Detailed descriptions of these weights and their calculation can be found in the technical reports on the study available at www.ifs.org.uk/elsa.

\section{Results}

Table 1 shows the baseline characteristics of the 1,726 men and women in the study according to whether they were not frail, pre-frail or frail at follow-up. The weighted percentage of participants who were pre-frail or frail at follow-up was 43 and $13 \%$, respectively. Mean Framingham CVD risk score at baseline rose with increasing degree of frailty at follow-up and differed significantly between those who were not frail, pre-frail or frail. As regards to the components of the Framingham CVD risk score, men and women who were older or who had diabetes at baseline tended to have a higher degree of frailty at follow-up. People who were pre-frail had higher systolic blood pressure at baseline than those who were not frail, but there were no differences in blood pressure between those who were pre-frail and those who were frail. Compared to those who were not frail at follow-up, those who were pre-frail were slightly more likely to have been a smoker at baseline, but this was of borderline significance, and there were no differences in baseline smoking status between those who were pre-frail and those who were frail at follow-up. Blood levels of total or HDL cholesterol and use of antihypertensive medication at baseline did not vary significantly by frailty status at follow-up. As regards to the covariates, cognitive function was poorer, level of household wealth lower and BMI higher at baseline in those with a greater degree of frailty at follow-up. There was no difference in sex distribution between those who were not frail and those who were pre-frail at follow-up,

Table 1 Baseline characteristics of the study participants $(n=1,726)$ according to frailty status at follow-up

\begin{tabular}{|c|c|c|c|c|c|}
\hline & $\begin{array}{l}\text { Not frail } \\
(n=862)\end{array}$ & $\begin{array}{l}\text { Pre-frail } \\
(n=691)\end{array}$ & $\begin{array}{l}\text { Frail } \\
(n=173)\end{array}$ & $\begin{array}{l}\text { P for difference } \\
\text { between not frail } \\
\text { and pre-frail }\end{array}$ & $\begin{array}{l}\mathrm{P} \text { for difference } \\
\text { between pre-frail } \\
\text { and frail }\end{array}$ \\
\hline Framingham CVD risk score, mean (SD) & $14.3(2.59)$ & $15.3(2.48)$ & $16.1(2.35)$ & $<0.0001$ & $<0.0001$ \\
\hline \multicolumn{6}{|l|}{ Framingham CVD risk score components } \\
\hline Age (years), mean (SD) & $67.1(6.20)$ & $71.6(7.33)$ & $76.2(7.83)$ & $<0.0001$ & 0.005 \\
\hline HDL cholesterol (mg/dl), mean (SD) & $61.0(16.1)$ & $60.1(15.0)$ & $60.7(14.3)$ & 0.261 & 0.267 \\
\hline Total cholesterol (mg/dl), mean (SD) & $236.8(50.2)$ & $236.6(46.2)$ & $235.9(47.1)$ & 0.838 & 0.705 \\
\hline Current smoker, $\%$ & 7.40 & 9.95 & 12.9 & 0.086 & 0.334 \\
\hline Systolic blood pressure (mm Hg), mean (SD) & $135.9(19.9)$ & $139.5(19.0)$ & $141.2(19.0)$ & 0.001 & 0.465 \\
\hline On anti-hypertensive medication, $\%$ & 13.7 & 16.0 & 15.5 & 0.238 & 0.882 \\
\hline Diabetes, $\%$ & 5.99 & 7.16 & 16.6 & 0.001 & 0.004 \\
\hline \multicolumn{6}{|l|}{ Covariates } \\
\hline Female, \% & 55.1 & 56.6 & 67.7 & 0.573 & 0.012 \\
\hline Cognitive function, mean (SD) & $30.2(5.74)$ & $27.5(5.52)$ & $24.7(4.96)$ & $<0.0001$ & $<0.0001$ \\
\hline Lowest quintile of household wealth, \% & 9.09 & 18.5 & 29.4 & $<0.0001$ & $<0.0001$ \\
\hline BMI $\left(\mathrm{kg} / \mathrm{m}^{2}\right)$, mean $(\mathrm{SD})$ & $27.2(4.24)$ & $27.5(4.39)$ & $28.7(5.24)$ & 0.031 & 0.006 \\
\hline No of frailty criteria present, mean (SD) & $0.29(0.65)$ & $0.79(0.85)$ & $1.65(1.14)$ & $<0.0001$ & $<0.0001$ \\
\hline
\end{tabular}


but there was a significantly higher proportion of women among those who were frail.

Preliminary analyses showed that the relationship between Framingham CVD risk score and risk of incident pre-frailty or frailty did not differ between the sexes ( $p$ for interaction term $>0.5$ ). Multivariate multinomial logistic regression analyses of frailty risk were therefore carried out in men and women together, adjusting for sex.

Table 2 shows relative risks for incident pre-frailty and frailty for a standard deviation increase in Framingham CVD risk score. Results are shown adjusted first for sex and baseline frailty status, and then with further adjustment for the other potential confounding variables, household wealth, cognitive function and BMI. In models adjusting for sex and baseline frailty status, there was a significant positive association between Framingham CVD risk score at baseline and risk of incident frailty: For a standard deviation increase in the Framingham risk score, the relative risk ratio (RR) (95\% confidence interval) for incident frailty was 2.76 $(2.18,3.49)$. Further adjustment for cognitive function, household wealth and BMI at baseline only partially attenuated the association: RR (95\% CI) 2.15 (1.68, 2.75). There was a significant association between total score for Framingham CVD risk score at baseline and risk of incident pre-frailty: For a standard deviation increase in the Framingham risk score, the relative risk ratio (95\% confidence interval) for incident pre-frailty was $1.69(1.46,1.95)$. Further adjustment for cognitive function, household wealth and BMI at baseline had only minor attenuating effects: RR (95\% CI) 1.50 (1.29, 1.74).

None of the participants in our sample had a reported history of CVD at baseline. To examine whether the associations between baseline Framingham CVD risk score and incident frailty or pre-frailty were concentrated among those who reported that they had been newly diagnosed with CVD during the 4-year follow-up period, we repeated our analysis after excluding this group $(n=68)$. Results were essentially unchanged.

In a subset analysis of the women in our sample $(n=982)$, we examined whether concentrations of the inflammatory markers fibrinogen and C-reactive protein-previously shown to be predictive of frailty in women only (Gale et al. 2013) -were confounders of the associations between baseline Framingham CVD risk score and incident frailty or pre-frailty. Adjustment for these biomarkers had little attenuating effect. For an SD increase in Framingham CVD score, the relative risk ratios for incident pre-frailty or frailty in women, adjusted for baseline frailty status, were $1.63(1.34,1.98)$ and 2.51 (1.87, 3.38), respectively. After further adjustment for baseline fibrinogen and C-reactive protein levels, these relative risk ratios were $1.61(1.32,1.96)$ and $2.34(1.73,3.18)$, respectively.

In order to investigate whether the associations observed between the Framingham CVD score at baseline and risk of incident frailty or pre-frailty were driven by particular components of the score (age, systolic blood pressure, use of anti-hypertensive medication, current smoking, HDL and total cholesterol and diabetes), we examined the effect on the sex and baseline frailty statusadjusted relative risks of further adjustment for each component in turn. The associations between CVD risk score and risk of pre-frailty and frailty were in almost all cases unattenuated by these adjustments (Table 3). The largest attenuating effects occurred when the associations between Framingham CVD risk score and risk of prefrailty or frailty were adjusted for age, but they remained statistically significant: Relative risk ratios for pre-frailty or frailty changed from $1.69(1.46,1.95)$ and $2.76(2.18$, $3.49)$ to $1.24(1.05,1.46)$ and $1.71(1.31,2.24)$, respectively. Adjustment for diabetes, the only component of the Framingham risk score apart from age that was consistently and strongly linked with frailty status (see Table 1), had no attenuating effect on the associations between CVD risk score and risk of pre-frailty and frailty.

Table 2 Relative risk ratios (95\% CI) for incident pre-frailty or frailty for a standard deviation increase in Framingham CVD risk score at baseline

\begin{tabular}{|c|c|c|c|c|}
\hline & \multicolumn{2}{|c|}{$\begin{array}{l}\mathrm{RR}(95 \% \mathrm{CI}) \text {, adjusted for sex and } \\
\text { baseline frailty status }\end{array}$} & \multicolumn{2}{|c|}{ RR $\left(95 \%\right.$ CI), fully adjusted ${ }^{1}$} \\
\hline & Pre-frailty & Frailty & Pre-frailty & Frailty \\
\hline Framingham CVD risk score, per SD increase & $1.69(1.46,1.95)$. & $2.76(2.18,3.49)$ & $1.50(1.29,1.74)$ & $2.15(1.68,2.75)$ \\
\hline
\end{tabular}

${ }^{1}$ Adjusted for sex, household wealth, cognitive function, BMI, and whether non-frail, pre-frail or frail at baseline 
Table 3 Relative risk ratios (95\% CI) for incident pre-frailty or frailty for a standard deviation increase in Framingham CVD risk score at baseline, separately adjusted for each component of the score

\begin{tabular}{lll}
\hline Framingham CVD risk score, per SD increase & \multicolumn{2}{l}{$\mathrm{RR}(95 \% \mathrm{CI}$} \\
\cline { 2 - 3 } & Pre-frailty & Frailty \\
\hline Adjusted for sex and baseline frailty status & $1.69(1.46,1.95)$ & $2.76(2.18,3.49)$ \\
Further adjusted for: & & \\
Age & $1.24(1.05,1.46)$ & $1.71(1.31,2.24)$ \\
Total cholesterol & $1.74(1.50,2.02)$ & $2.89(2.28,3.67)$ \\
HDL cholesterol & $1.68(1.45,1.84)$ & $2.87(2.25,3.67)$ \\
Systolic blood pressure & $1.63(1.46,1.95)$ & $2.62(2.07,3.33)$ \\
Anti-hypertensive treatment & $1.65(1.45,1.87)$ & $2.76(2.18,3.49)$ \\
Smoking & $1.69(1.46,1.95)$ & $3.13(2.43,4.04)$ \\
Diabetes & $1.80(1.53,2.11)$ & $2.77(2.12,3.61)$ \\
\hline
\end{tabular}

To explore whether the associations might be better explained by a continuous measure of glucose metabolism, we adjusted for levels of haemoglobin A1C, but this too had no attenuating effect (data not shown). These results suggest that no specific component risk factor drives the associations between Framingham CVD risk score and risk of pre-frailty or frailty.

\section{Discussion}

In this prospective study of men and women aged 60 to over 90 years, none of whom had a history of CVD, higher Framingham CVD risk scores at baseline were associated with an increased risk of incident frailty and pre-frailty. Adjustment for potential confounding factors had only small attenuating effects on these associations, and they were little changed by the exclusion of those who developed CVD during follow-up.

To our knowledge, only one previous study has examined the relation between Framingham CVD risk scores and subsequent frailty. In the Whitehall II study, an SD increase in Framingham CVD score in people aged 45-69 years increased the odds of being frail 10 years later by $42 \%$ (Bouillon et al. 2013). As in the present study, no single component of the CVD risk score appeared to be driving its association with future frailty. Direct comparison of effect sizes with those found in our study is difficult because we used multinomial rather than binary logistic regression so that we could examine whether Framingham CVD score was linked with risk of pre-frailty as well as frailty, but our findings suggest that the risk of frailty associated with raised Framingham CVD scores in older people is at least as high and possibly higher than that observed in middle-aged individuals. Furthermore, our results suggest that these CVD scores are also predictive of risk of pre-frailty-defined by Fried as the presence of just one or two of the five components of frailty (Fried et al. 2001). This has potential implications for prevention or amelioration. Whereas transitions to a state of greater frailty are more common than movement in the reverse direction, there is sufficient evidence of the latter to suggest that frailty is potentially reversible, at least in the less severe stage (Espinoza et al. 2012; Gill et al. 2006).

Several mechanisms could potentially underlie the association we observed between Framingham CVD risk scores in people with no known history of CVD and later risk of frailty. One possibility is that atherosclerosis affects blood flow to the nerves and muscles of the legs, exacerbating sarcopenia, a major component of frailty (Morley et al. 2002). There is some evidence that the prevalence of frailty is higher in older people with peripheral atherosclerosis as indicated by a high anklebrachial index or other indicators of subclinical CVD such as carotid stenosis or left ventricular hypertrophy (Newman et al. 2001; Singh et al. 2012). In the Three Cities study of older people, mean walking speed decreased with increasing number of carotid plaques and increased intimal-medial thickening (Elbaz et al. 2005). Another related factor might be brain pathology. Brain autopsy in members of two US cohorts - the Religious Orders Study and the Rush Memory and Aging Project-followed for up to 14 years showed that the presence of subclinical CVD and Alzheimer pathologies was associated with more rapid progression of frailty, and in particular with more rapid decline in walking speed (Buchman et al. 2013). Although inflammation 
is a plausible underlying mechanism, given observations in several studies linking higher levels of inflammatory biomarkers with higher CVD risk scores (Noh et al. 2013; Park et al. 2010) and increased risk of incident frailty (Baylis et al. 2012; Puts et al. 2005), we found little evidence that it played a part in the associations we found. Another potential underlying mechanism is impaired glucose metabolism. There is evidence that muscle strength and physical function are poorer in older people with raised glucose levels, and this is not confined to those with diabetes (Sayer et al. 2005). Diabetes has been linked with increased risk of becoming frail (Lee et al. 2014), perhaps by accelerating age-related muscle loss (Kalyani et al. 2014). However, in this study, although the presence of diabetes at baseline was strongly associated with greater frailty at follow-up, neither it nor levels of a baseline marker of glucose metabolism - haemoglobin A1C - helped to explain the association between Framingham CVD risk scores and incident pre-frailty or frailty.

The strengths of our study include the large sample size, the fact that ELSA was designed to be representative of the older community-dwelling English population (Steptoe et al. 2013), and our use of a prospective study design in which we assessed incident cases of prefrailty or frailty that occurred after the measurement of CVD risk factors. There are also some weaknesses. First, information on prevalent and incident CVD was based solely on reported doctor diagnoses of angina, heart attack, congestive heart failure or stroke. Second, not all the participants in the baseline survey gave a blood sample (77\% of those interviewed); nonresponders to the blood sample tended to be older and in poorer health. However, in view of the fact that the associations we found between Framingham CVD risk score and risk of frailty were at least as strong as those reported in a previous study of middle-aged people (Bouillon et al. 2013), it seems unlikely that our findings would be very different if we had validated data on history of CVD and complete data on blood biomarkers for all those who were interviewed at baseline. Finally, our follow-up period was relatively short-around 4 years - which raises the possibility of reverse causation whereby the higher baseline Framingham CVD risk scores observed in people who were frail or pre-frail at follow-up might be a consequence of their prior frailty status. We can be fairly sure that this is not the case as the association we found was robust to adjustment for the number of components of frailty present at baseline.
Furthermore, it is consistent with observations from other longitudinal studies with much longer follow-up times that higher levels of cardiovascular risk factors in midlife - whether assessed as overweight or obesity (Stenholm et al. 2014), physical activity (Savela et al. 2013) or a composite risk factor score for coronary artery disease (Strandberg et al. 2012) —increase the risk of frailty over 20 years later.

In this prospective study of men and women aged 60 to over 90 years, none of whom had a history of CVD; higher Framingham CVD risk scores were associated with an increased risk of incident frailty and pre-frailty. Use of these scores in clinical practice may help identify those older people who are at risk of becoming frail. We now need to understand the biological mechanisms whereby CVD risk increases the risk of frailty.

Acknowledgments The data were made available through the UK Data Archive. ELSA was developed by a team of researchers based at the National Centre for Social Research, University College London and the Institute for Fiscal Studies. The data were collected by the National Centre for Social Research. The funding is provided by the National Institute of Aging in the United States, and a consortium of UK government departments coordinated by the Office for National Statistics. The developers and funders of ELSA and the Archive do not bear any responsibility for the analyses or interpretations presented here. This work was supported by the Medical Research Council (MRC_MC_UU_12011/2 and MRC_MC_UP_A620_1015).

Open Access This article is distributed under the terms of the Creative Commons Attribution License which permits any use, distribution, and reproduction in any medium, provided the original author(s) and the source are credited.

\section{References}

Afilalo J, Karunananthan S, Eisenberg MJ, Alexander KP, Bergman H (2009) Role of frailty in patients with cardiovascular disease. Am J Cardiol 103:1616-1621. doi:10.1016/j. amjcard.2009.01.375

Afilalo J, Alexander KP, Mack MJ, Maurer MS, Green P, Allen LA, Popma JJ, Ferrucci L, Forman DE (2014) Frailty assessment in the cardiovascular care of older adults. J Am Coll Cardiol 63:747-762. doi:10.1016/j.jacc.2013.09.070

Bandeen-Roche K, Xue QL, Ferrucci L, Walston J, Guralnik JM, Chaves P, Zeger SL, Fried LP (2006) Phenotype of frailty: characterization in the women's health and aging studies. $J$ Gerontol A Biol Sci Med Sci 61:262-266

Banks J, Karlsen S, Oldfield Z (2003) Socio-economic position. In: Marmot M, Banks J, Blundell R, Lessof C, Nazroo J (eds) Health, wealth and lifestyles of the older population in England. Institute of Fiscal Studies, London, pp 71-125 
Baylis D, Bartlett DB, Syddall HE, Ntani G, Gale CR, Cooper C, Lord JM, Sayer AA (2012) Immune-endocrine biomarkers as predictors of frailty and mortality: a 10-year longitudinal study in community-dwelling older people. Age (Dordr). doi:10.1007/s11357-012-9396-8

Bergman H, Ferrucci L, Guralnik J, Hogan DB, Hummel S, Karunananthan S, Wolfson C (2007) Frailty: an emerging research and clinical paradigm-issues and controversies. J Gerontol A Biol Sci Med Sci 62:731-737

Bouillon K, Batty GD, Hamer M, Sabia S, Shipley MJ, Britton A, Singh-Manoux A, Kivimaki M (2013) Cardiovascular disease risk scores in identifying future frailty: the Whitehall II prospective cohort study. Heart 99:737-742. doi:10.1136/ heartjnl-2012-302922

Brayne C, Nickson J, McCracken C, Gill C, Johnson AL (1998) Cognitive function and dementia in six areas of England and Wales: the distribution of MMSE and prevalence of GMS organicity level in the MRC CFA study. Psychol Med 28: 319-335

Buchman AS, Yu L, Wilson RS, Schneider JA, Bennett DA (2013) Association of brain pathology with the progression of frailty in older adults. Neurology 80:2055-2061. doi:10.1212/ WNL.0b013e318294b462

Clegg A, Young J, Iliffe S, Rikkert MO, Rockwood K (2013) Frailty in elderly people. Lancet 381:752-762. doi:10.1016/ S0140-6736(12)62167-9

D'Agostino RB Sr, Vasan RS, Pencina MJ, Wolf PA, Cobain M, Massaro JM, Kannel WB (2008) General cardiovascular risk profile for use in primary care: the Framingham Heart Study. Circulation 117:743-753. doi:10.1161/CIRCULATIONAHA. 107.699579

Elbaz A, Ripert M, Tavernier B, Fevrier B, Zureik M, Gariepy J, Alperovitch A, Tzourio C (2005) Common carotid artery intimamedia thickness, carotid plaques, and walking speed. Stroke 36: 2198-2202. doi:10.1161/01.STR.0000181752.16915.5c

Elbaz A, Shipley MJ, Nabi H, Brunner EJ, Kivimaki M, SinghManoux A (2014) Trajectories of the Framingham general cardiovascular risk profile in midlife and poor motor function later in life: the Whitehall II study. Int J Cardiol 172:96-102. doi:10.1016/j.ijcard.2013.12.051

Espinoza SE, Jung I, Hazuda H (2012) Frailty transitions in the San Antonio Longitudinal Study of Aging. J Am Geriatr Soc 60:652-660. doi:10.1111/j.1532-5415.2011.03882.x

Fried LP, Tangen CM, Walston J, Newman AB, Hirsch C, Gottdiener J, Seeman T, Tracy R, Kop WJ, Burke G, McBurnie MA (2001) Frailty in older adults: evidence for a phenotype. J Gerontol A Biol Sci Med Sci 56:M146-M156

Gale CR, Baylis D, Cooper C, Sayer AA (2013) Inflammatory markers and incident frailty in men and women: the English Longitudinal Study of Ageing. Age 35:2493-2501. doi:10. 1007/s11357-013-9528-9

Gale CR, Cooper C, Aihie Sayer A (2014a) Prevalence of frailty and disability: findings from the English Longitudinal Study of Ageing. Age Ageing, in press

Gale CR, Cooper C, Deary IJ, Aihie Sayer A (2014b) Psychological well-being and incident frailty in men and women: the English Longitudinal Study of Ageing. Psychol Med 44:697-706. doi:10.1017/S0033291713001384

Gill TM, Gahbauer EA, Allore HG, Han L (2006) Transitions between frailty states among community-living older persons. Arch Intern Med 166:418-423. doi:10.1001/.418
Kaffashian S, Dugravot A, Elbaz A, Shipley MJ, Sabia S, Kivimaki M, Singh-Manoux A (2013) Predicting cognitive decline: a dementia risk score vs. the Framingham vascular risk scores. Neurology 80:1300-1306. doi:10.1212/WNL. 0b013e31828ab370

Kalyani RR, Corriere M, Ferrucci L (2014) Age-related and disease-related muscle loss: the effect of diabetes, obesity, and other diseases. Lancet Diabetes Endocrinol. doi:10.1016/ S2213-8587(14)70034-8

Lee JS, Auyeung TW, Leung J, Kwok T, Woo J (2014) Transitions in frailty states among community-living older adults and their associated factors. J Am Med Dir Assoc 15:281-286. doi:10.1016/j.jamda.2013.12.002

Marmot M, Oldfield Z, Clemens S, Blake M, Phelps A, Nazroo J, Steptoe A, Rogers N, Bank J (2013) English Longitudinal Study of Ageing: waves 0-5, 1998-2011 [computer file], 20th edn. SN: 5050. UK Data Archive [distributor], Colchester

Morley JE, Perry HM 3rd, Miller DK (2002) Editorial: something about frailty. J Gerontol A Biol Sci Med Sci 57:M698-M704

Newman AB, Gottdiener JS, McBurnie MA, Hirsch CH, Kop WJ, Tracy R, Walston JD, Fried LP, Cardiovascular Health Study Research G (2001) Associations of subclinical cardiovascular disease with frailty. J Gerontol A Biol Sci Med Sci 56:M158M166

Noh TS, Moon SH, Cho YS, Hong SP, Lee EJ, Choi JY, Kim BT, Lee KH (2013) Relation of carotid artery 18 F-FDG uptake to $\mathrm{C}$-reactive protein and Framingham risk score in a large cohort of asymptomatic adults. J Nucl Med: Off Publ, Soc Nucl Med 54:2070-2076. doi:10.2967/jnumed.113.119602

Ofstedal M, Fisher G, Hertzog R (2005) Documentation of cognitive functioning measures in the Health and Retirement Study. http://hrsonline.isr.umich.edu/sitedocs/userq/dr-006. pdf. Accessed February 2014

Park CS, Ihm SH, Yoo KD, Kim DB, Lee JM, Kim HY, Chung WS, Seung KB, Kim JH (2010) Relation between C-reactive protein, homocysteine levels, fibrinogen, and lipoprotein levels and leukocyte and platelet counts, and 10-year risk for cardiovascular disease among healthy adults in the USA. Am J Cardiol 105:1284-1288. doi:10.1016/j.amjcard.2009.12.045

Puts MT, Visser M, Twisk JW, Deeg DJ, Lips P (2005) Endocrine and inflammatory markers as predictors of frailty. Clin Endocrinol (Oxf) 63:403-411. doi:10.1111/j.1365-2265. 2005.02355.x

Rockwood K, Fox RA, Stolee P, Robertson D, Beattie BL (1994) Frailty in elderly people: an evolving concept. Can Med Assoc J 150:489-495

Roth M, Huppert FA, Mountjoy CQ, Tym E (1999) The revised Cambridge examination for mental disorders of the elderly. Cambridge University Press, Cambridge

Savela SL, Koistinen P, Stenholm S, Tilvis RS, Strandberg AY, Pitkala KH, Salomaa VV, Strandberg TE (2013) Leisure-time physical activity in midlife is related to old age frailty. J Gerontol A Biol Sci Med Sci 68:1433-1438. doi:10.1093/ gerona/glt029

Sayer AA, Dennison EM, Syddall HE, Gilbody HJ, Phillips DI, Cooper C (2005) Type 2 diabetes, muscle strength, and impaired physical function: the tip of the iceberg? Diabetes Care 28:2541-2542

Singh S, Bailey KR, Noheria A, Kullo IJ (2012) Frailty across the spectrum of ankle-brachial index. Angiology 63:229-236. doi:10.1177/0003319711413457 
Steffick DE, The HRS working group (2000) Documentation of affective functioning measures in the Health and Retirement Study. HRS/AHEAD Documentation Report DR-005 [online report]. http://hrsonline.isr.umich.edu/ sitedocs/userg/dr-005.pdf

Stenholm S, Strandberg TE, Pitkala K, Sainio P, Heliovaara M, Koskinen S (2014) Midlife obesity and risk of frailty in old age during a 22-year follow-up in men and women: the MiniFinland Follow-up Survey. J Gerontol A Biol Sci Med Sci 69:73-78

Steptoe A, Breeze E, Banks J, Nazroo J (2013) Cohort profile: the English Longitudinal Study of Ageing. Int J Epidemiol 42: 1640-1648. doi:10.1093/ije/dys168

Strandberg TE, Sirola J, Pitkala KH, Tilvis RS, Strandberg AY, Stenholm S (2012) Association of midlife obesity and cardiovascular risk with old age frailty: a 26-year follow-up of initially healthy men. Int J Obes (Lond) 36:1153-1157. doi: 10.1038/ijo.2012.83
The International Expert Committee (2009) International Expert Committee Report on the role of the A1C assay in the diagnosis of diabetes. Diabetes Care 32:1-8

von Haehling S, Anker SD, Doehner W, Morley JE, Vellas B (2013) Frailty and heart disease. Int J Cardiol 168:17451747. doi:10.1016/j.ijcard.2013.07.068

Walston J, Hadley EC, Ferrucci L, Guralnik JM, Newman AB, Studenski SA, Ershler WB, Harris T, Fried LP (2006) Research agenda for frailty in older adults: toward a better understanding of physiology and etiology: summary from the American Geriatrics Society/National Institute on Aging Research Conference on Frailty in Older Adults. J Am Geriatr Soc 54:991-1001

Wong CH, Weiss D, Sourial N, Karunananthan S, Quail JM, Wolfson C, Bergman H (2010) Frailty and its association with disability and comorbidity in a community-dwelling sample of seniors in Montreal: a cross-sectional study. Aging Clin Exp Res 22:54-62. doi:10.3275/6675 